\title{
Propuesta de aplicación de la metodología PMBOK para un proyecto de construcción de 20 casas ecológicas en condominio en zona rural de Palmira (Corregimiento La Zapata)
}

\author{
Julio Cesar Montoya R. ${ }^{1}$ \\ Oscar Eduardo Sanclemente ${ }^{2}$ \\ Alicia Cristian Silva C. ${ }^{3}$
}

\section{RESUMEN}

El presente documento investigativo, tuvo como objeto de estudio, una propuesta de índole eco ambiental, mediante el cual se pretende caracterizar la construcción de un condominio de casas ecológicas en la vereda El Agrado, jurisdicción del Corregimiento de La Zapata, bajo la guía de los fundamentos de la dirección de proyectos más conocida como PMBOK.

La importancia del PMBOK es que provee un marco de referencia formal para desarrollar proyectos, guiando y orientando a los gerentes sobre la forma de avanzar en los procesos y los pasos necesarios para la consolidación de un proyecto en forma detallada.

La estructura del documento como tal, inicia con los antecedentes de este tipo de construcciones en Colombia, y su relevancia en el gremio industrial local,

Las fases subsiguientes, completan la secuencia lógica de actividades que conforman la estructura de la guía PMBOK; es decir el paso a paso de la Administración del proyecto que incluye; el acta de constitución del proyecto, la declaración del alcance del proyecto, el registro de interesados, el plan de gestión del proyecto con todos sus subplanes, y finaliza con el cierre del proyecto.

\footnotetext{
${ }_{1}^{1}$ Administrador de Empresas, Especialista en Pedagogía para el Desarrollo del Aprendizaje Autónomo, Maestría en Administración de Empresas.Docente de la Universidad Nacional Abierta y a Distancia UNAD. julio.montoya@unad.edu.co

2 Ingeniero Ambiental, Magister en Ciencias Agrarias, Doctor en Agroecología, Docente Universidad Nacional Abierta y a Distancia - UNAD. oscar.sanclemente@unad.edu.co

${ }^{3}$ Administradora de empresas, especialista en Pedagogía para el Desarrollo del Aprendizaje Autónomo. Magíster en Sistemas de Calidad y Productividad.Estudiante de Doctorado de Administración de Negocios, mención Gerencia. Docente Universidad Nacional Abierta y Distancia - UNAD. alicia.silva@unad.edu.co.
} 
Palabras claves: gestión, proyecto, recursos, alcance, interesados

\section{Introducción}

La construcción de las "casas ecológicas" es un tema que ha venido cogiendo fuerza debido a la proliferación de campañas que promueven las tecnologías limpias que garantizan un desarrollo sostenible. El valor agregado de este tipo de viviendas es que debido a los elementos que tiene, desde su estructura, hasta con los que funciona, contribuyen eficientemente al medio ambiente, mejorando la calidad del aire, no usando y gastando combustibles fósiles, reciclando, entre otras cosas.

No obstante, la gran valía de este tipo de edificaciones es su bajo costo tanto en la construcción, como en su mantenimiento, existen incluso talleres donde a moderadas tarifas cualquier persona puede adquirir competencias para edificar esta clase de viviendas.

La presente investigación, describe el paso a paso de la aplicación de la guía metodológica PMBOK para implementar un proyecto de construcción de 20 casas ecológicas en zona rural del municipio de Palmira. La pretensión de este ejercicio investigativo es seguir fielmente la estructura secuencial del PMBOK para garantizar la idoneidad del proyecto.

\section{Antecedentes}

Los referentes que a continuación se relacionan, obedecen a documentos que por su pertinencia con el tema objeto de estudio, posibilitan una mayor comprensión del mismo, además que sustentan la relevancia de la aplicación de la guía metodología PMBOK.

El primer referente corresponde a un trabajo de grado desarrollado para la Universidad Nacional Mayor de San Marcos (Lima, Perú), titulado "Aplicación de la guía del pmbok $\AA$ en el desarrollo de nuevos productos farmacéuticos en un área de Investigación y Desarrollo" (Soto Vicente, 2015). En este trabajo fueron aplicados los conceptos y metodologías de la guía del Instituto de Gestión de Proyectos (Project Management Institute - Guía del PMBOK®) y conceptos de administración como outsorcing, benchmarking y empowerment en el desarrollo de nuevos productos farmacéuticos a fin de incrementar la competitividad, efectividad, reducción de riesgos, optimizar los tiempos y éxito en el desarrollo asegurando su seguridad y eficacia de acuerdo a los parámetros internacionales de calidad y salud pública requeridas.

La contribución de este documento es interesante, pues su autor no solo aplica las herramientas metodológicas de esta guía, sino que se aventura a predecir los beneficios de su implementación en variables como ahorro en dinero y tiempo, lo que en realidad se cumplió al observar los resultados cuantitativos de dicha investigación. 
Un segundo referente de singular relevancia por su enfoque práctico, es el desarrollado con el nombre de "El proyecto del coche eléctrico en España. La gestión basada en PMBOK®" ( (López Doval, 2012). Esta investigación tuvo como finalidad investigar y generar conocimiento en los temas clave necesarios para la fabricación y comercialización de vehículos ecológicos en España, lo que permitiría potencialmente reducir la dependencia energética del petróleo de este país, reducir las emisiones de CO2 en el sector del transporte, favoreciendo la penetración de las energías renovables, y garantizar el futuro del sector industrial y del I+D de la automoción en España. Como bien puede apreciar es un proyecto muy ambicioso que requería de tomar todos los recaudos necesarios para garantizar su éxito.

Este macroproyecto al menos como quedó planteado utilizando la guía PMBOK, tiene todas las posibilidades de garantizar excelentes réditos, teniendo en cuenta que se completaron todos los ítems de esta guía. Esto sirve para confirmar que la guía PMBOK ha sido dispuesta para macros y micros proyectos por igual, y que necesariamente deben cumplirse cada uno de sus apartados.

Un referente muy pertinente, corresponde al trabajo de grado "integración entre PSP y PMBOK® aplicada al desarrollo de un sistema experto para el diagnóstico e identificación automática de enfermedades profesionales" (Ramirez Zuluaga, 2011). Este documento El presente trabajo enseña un subconjunto de buenas prácticas tanto del proceso de desarrollo de software a nivel individual con PSP (Personal Software Process) como la guía para la gerencia de proyectos definida en el marco de referencia de PMBOK® (Project Management Body of Knowledge) para ayudar a gestionar con efectividad un proceso de desarrollo de software con estándares y guías.

\section{Aplicación de la Guía PMOBK al proyecto KAEKO}

En este apartado, se procedió a aplicar la guía metodológica PMBOK al proyecto KAEKO (Casas ecológicas)

\section{Gestión de la integración del proyecto}

\section{Desarrollo del acta de constitución del proyecto}

En la tabla $\mathrm{N}^{\circ} 1$ se describe los componentes del acta de constitución del proyecto.

Tabla 1. Acta de constitución del proyecto

\begin{tabular}{ll}
\hline TITULO DEL PROYECTO & $\begin{array}{l}\text { Construcción de } 20 \text { casas ecológicas en } \\
\text { zona rural del municipio de Palmira }\end{array}$ \\
\hline CODIGO & PK-001 \\
FECHA & $04 / 01 / 2016$
\end{tabular}


GERENTE DEL PROYECTO

PATROCINADOR DEL PROYECTO

1.Descripción del proyecto

La intencionalidad de desarrollar este proyecto es la construcción de 30 viviendas de tipo ecológico en zona rural del municipio de Palmira. Es un proyecto que propende por sentar un precedente positivo en la comunidad en cuanto a la conservación del medio ambiente, puesto que la idea con el mismo, es utilizar material ciento por ciento autosostenible. El proyecto comprende desde la aprobación del mismo por parte de las entidades estatales y ambientales hasta la culminación de la obra, y estará financiado por capital privado de una familia muy arraigada a esta región.

2. Justificación del proyecto

La razón de ser de este proyecto, reside en la promoción y consolidación de la bioarquitectura, como alternativa idónea para contribuir al medio ambiente, en una zona rural, donde por décadas, ha sido objeto de prácticas inadecuadas de manejo ecoambiental, como tala de bosques, excesiva desarenación de los ríos, y destrucción del hábitats naturales, entre otros. Otra razón no menos importante, es la generación de empleo o directo, como aporte social y económico a los vecinos y habitantes de esta comunidad veredal.

3. Localización del proyecto

El proyecto estará localizado en la vereda El Agrado, lugar que pertenece a la jurisdicción del corregimiento La Zapata, a $16 \mathrm{~km}$ hacia la zona montañosa oriental de Palmira.

4. Fecha de inicio

Marzo 29 de 2016

5. Duración del proyecto

8 meses

6. Dirección del proyecto

Consorcio KAEKO

7. Director del Proyecto

Arq. Samuel Arango Shima

8. Presupuesto

$\$ 600.000 .000$

9. Lista de Interesados (Stakeholders)

Stakeholders directos:

- IDEAM

- CVC

- Inderena

- Consejo Colombiano de Construcción Sostenible (CCCS)

- Alcaldía Municipal de Palmira

Stakeholders indirectos:

- Residentes de la vereda El Agrado

- Junta de acción comunal vereda El Agrado

- Proveedoras de guadua-bambú 
10. Objetivos del proyecto

10.1 Construir 20 viviendas ecológicas en sector rural del municipio de Palmira 10.2 Sentar un precedente de fomento para que se consolide este tipo construcciones teniendo en cuenta que propenden por la conservación del medio ambiente.

10.3 Cumplir con la planificación propuesta

11. Requisitos de alto nivel

- Que todos los materiales sean autosostenibles

- El tamaño del terreno.

- Que no existan cables de alta tensión en las cercanías ni industrias que contaminen.

- Buena orientación, ya sea que no existan barreras solares.

- Tipo de suelo.

- Conocer el microclima del lugar.

- Deben de analizar si hay fallas geológicas y venas de agua.

- Si existe contaminación química, eléctrica, magnética, ambiental y anímica.

12. Alcance del proyecto

- Permisos y licencias legales

- Construcción de 20 viviendas de acuerdo a especificaciones técnicas

- Gestión del Proyecto

- Transporte de materiales y equipos necesarios

Fuera del Alcance:

- Plan de manejo ambiental

- Negociaciones de personal de obra, con personas de la zona.

13. Riesgos del proyecto

- Tiempo y presupuesto mal calculado

- Roles y responsabilidades mal definidos

- Factores climáticos que retrasen los trabajos y/o logística de materiales y equipos

- Inadecuado seguimiento al movimiento logístico

- Robos de materiales y equipos

- Conflictos laborales con personal de la zona.

14. Restricciones del proyecto

Presupuesto: $\$ 600.000 .000$

Fecha de entrega: Noviembre 29 de 2016

15. Supuestos del proyecto

- Compromiso de la Dirección del proyecto

- Compromiso de todas las áreas involucradas

- Aprobación del proyecto por parte de las entidades competentes

- Cumplir con los plazos establecidos para obtener la Licencia de construcción 


\section{Gestión del alcance Del Proyecto}

La Gestión del Alcance del Proyecto incluye los procesos necesarios para garantizar que el proyecto incluya todo el trabajo requerido y únicamente el trabajo para completar el proyecto con éxito. Gestionar el alcance del proyecto se enfoca primordialmente en definir y controlar qué se incluye y qué no se incluye en el proyecto. (PMI, 2008)

\section{Factores ambientales de la empresa}

Los factores ambientales de la empresa que pueden influir en el proceso de Planificar la Gestión del Alcance incluyen, entre otros:

- La cultura de la organización: En el caso de la entidad proponente del proyecto, (Proyecto Kaeko) se puede aseverar que su fundamento es la ecosostenibilidad, lo que de antemano sesga todas sus actividades a dicha naturaleza, en tal sentido, este proyecto difiere sustancialmente a cualquier proyecto de construcción de viviendas de índole tradicional.

- La infraestructura: El conjunto de elementos o servicios considerados como necesarios para que esta organización pueda funcionar lo conforma un equipo reducido de profesionales como recurso humano, un capital privado, y recursos técnicos identificados con la ecosostenibilidad.

- La gestión de personal, obedece a las actividades a desarrollar por cada uno de los miembros del proyecto (roles, funciones, tareas, procedimientos), todo esto será formalmente establecido, porque se cuenta con gente capaz y con gran sentido de compromiso no solo con este proyecto sino con el medioambiente.

- Las condiciones del mercado. Respecto a este factor, el ambiente no puede ser más propicio, pues debido a la proliferación de prácticas limpias para la conservación del medio ambiente, proyectos como éste, son observados y apoyados con mucho optimismo.

\section{Activos de los procesos de la organización}

Los activos de los procesos de la organización son los planes, los procesos, las políticas, los procedimientos y las bases de conocimiento específicos de la organización ejecutora y utilizados por la misma. Estos incluyen cualquier objeto, práctica 0 conocimiento de alguna o de todas las organizaciones que participan en el proyecto y que pueden usarse para ejecutar o gobernar el proyecto. (PMI, 2008)

\section{Procesos y procedimientos}

Los procesos y procedimientos de la organización para realizar el trabajo del proyecto incluyen, entre otros:

- Inicio y Planificación:

Estándares específicos de la organización 
Para este proyecto específico se plantean las siguientes políticas:

\section{Políticas de Recursos Humanos}

$\checkmark$ Cada obrero y/o empleado tendrá su propia responsabilidad personal de acuerdo a sus competencias, pero con énfasis comunitario y solidario con sus pares.

$\checkmark$ Valores como la puntualidad, responsabilidad, compromiso deberán constituirse en mandatos indefectibles para sus labores.

$\checkmark$ Cualquier forma de intolerancia, de acoso o de discriminación será considerada como la expresión de una falta de respeto elemental.

$\checkmark$ Todo colaborador (empleado, obrero, oficial, ayudante, entre otros) deberá tener una actitud decidida y comprometida con el medio ambiente, en cada una de las tareas, roles y funciones asignadas.

$\checkmark$ El recurso humano escogido para este proyecto tendrá libertad de expresar sus opiniones de índole técnico, logístico, operativo y administrativo, siempre y cuando estén sujetas a los lineamientos y directrices del conducto regular pertinente, determinado por la Gerencia.

\section{Política de seguridad y salud}

$\checkmark$ KAEKO S.A.S desarrolla sus actividades de construcción ecosostenible promoviendo la protección a la Vida y a la Salud de sus empleados propios, contratistas, visitantes y partes interesadas. El compromiso de la Gerencia es implementar en la organización el SGSST (Sistema de Gestión de la Seguridad y Salud en el Trabajo), de conformidad al cumplimiento del Decreto 1443 de 2014.

\section{Política de ética}

$\checkmark$ La empresa se compromete a desarrollar prácticas limpias de índole jurídico, administrativo, logístico, comercial y operativo.

$\checkmark$ Los colaboradores de la empresa tendrán salarios justos conforme a sus competencias, destrezas y habilidades; a su vez tendrán programas de constante motivación para que su concurso individual al seno de la organización sea lo suficientemente honesto y transparente.

$\checkmark$ La interacción con los stakeholders de la organización (clientes, proveedores, aliados, inversionistas, socios, etc.) tendrá como lema la transparencia en el proceso comunicativo, con el fin de consolidar vínculos estables y de inobjetable pulcritud administrativa.

Para este proyecto específico se plantea el uso de la siguiente plantilla, para controlar la gestión de riegos (Figura 1) 
Figura 1. Plantilla control de riesgos

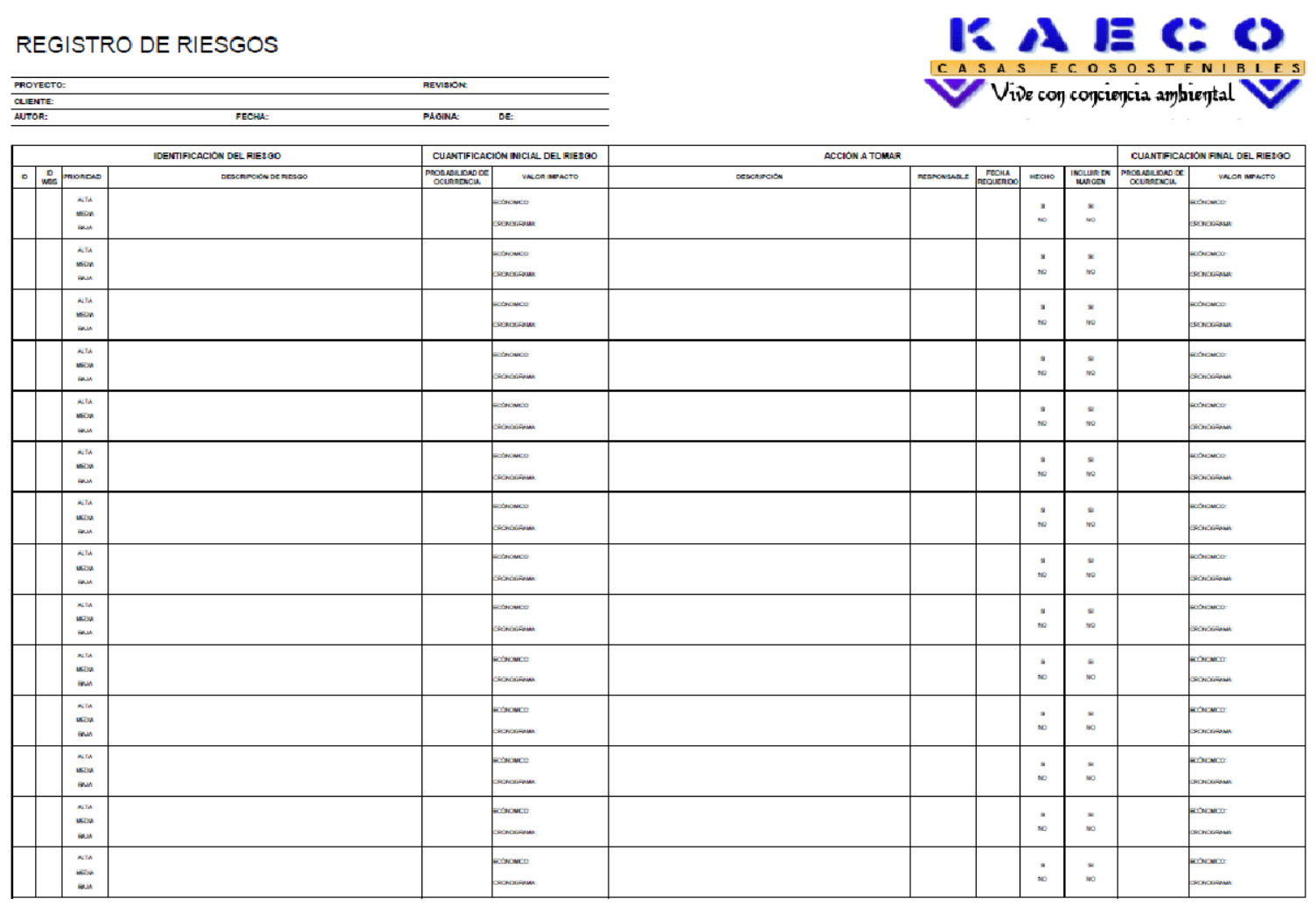

Fuente: Elaboración propia basada en el modelo PMOBK 


\section{EDT (Estructura desglose del Trabajo)}

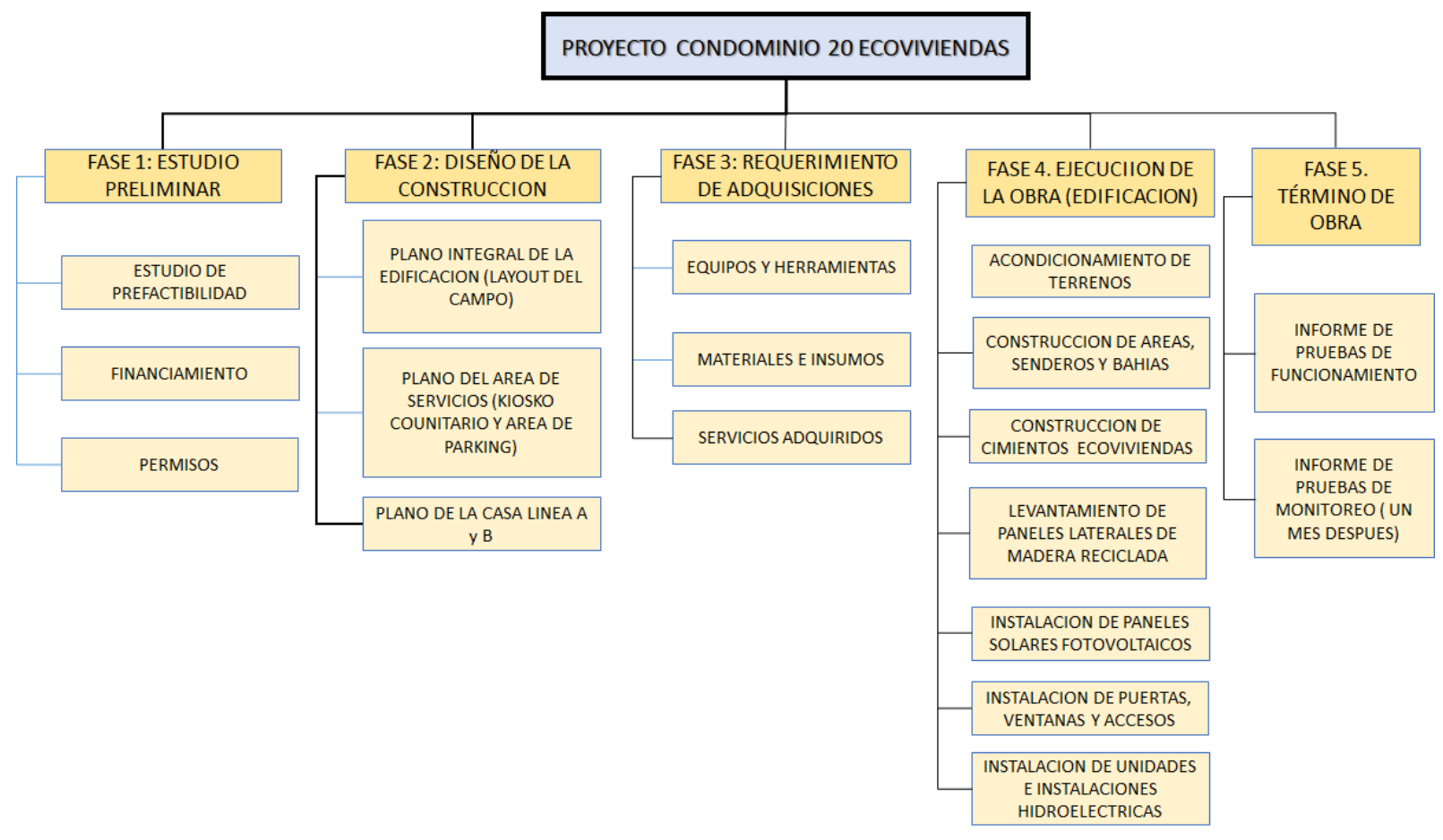

\section{Diccionario de la Estructura de Desglose de Trabajo (EDT)}

El diccionario de la EDT sustenta teóricamente la EDT (Estructura de Desglose de Trabajo), puesto que en éste documento, se entra en detalle sobre cada uno de los componentes de la misma, con base en los ítems y apartados que exige la guía metodología PMBOK, que en el caso particular del proyecto objeto de estudio (construcción de casas ecológicas o ecoviviendas) se hizo teniendo en cuenta las actividades más los entregables asociados.

\section{Cuadro 1. Diccionario de la EDT}

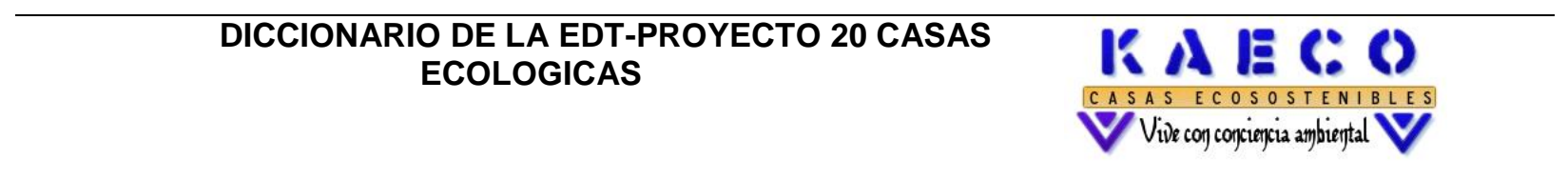

\section{PAQUETES DE TRABAJO}

Fase \# 1 Entregable: Estudio Preliminar

Fase \# 2 Entregable: Diseño de la construcción

Fase \# 3. Entregable: Requerimiento de adquisiciones

Fase \#4: Entregable: Ejecución de la obra (Edificación)

Fase \#5: Entregable: Término de obra 


\section{Descripción de los paquetes de trabajo}

Estudio Preliminar: La idea de este estudio previo, es evaluar en forma anticipada los diferentes eventos o circunstancias que puedan presentarse, y que por su relevancia es conveniente identificarlos, en este proceso también se involucran dos elementos claves para el desarrollo del proyecto; el financiamiento y los permisos a solicitar. Cabe recordar que el proyecto tiene una naturaleza ecológica, por ende, la consecución de los permisos ambientales resulta impostergable y definitiva.

Diseño de la Construcción: Refiere a los diferentes planos arquitectónicos que hay que levantar para el proyecto, en este caso, se necesitará un plano general, y dos planos individuales, el del área de servicios (bahías, kiosco comunitario, senderos) y el plano de las casas para ambas líneas.

Requerimiento de adquisiciones: Se requerirá realizar adquisiciones de materia prima, incluyendo los paneles solares fotovoltaicos, que producirán la energía para la casa; de igual forma, se deberá comprar madera, guadua, arena, balastro, cemento, los vidrios para las ventanas, herramientas, la palmicha para parte del techo, y el generador fotovoltaico, que es lo más costoso. Aunado a la compra de materia prima, es necesario contratar la mano de obra, y otros servicios como buldozer para la adecuación e igualación de los terrenos.

Ejecución de la obra: Se realizará la construcción de las diferentes áreas que conforman el proyecto: área de servicios (kiosko comunitario, senderos, bahías), y la construcción del condominio). Para cada una de estas áreas, un entregable debe asociarse:

Área de servicios:

- Cimientos del Kiosco

- Entablado

- Colocación de adoquines para los senderos

- Vallado en madera

- Postes de iluminación

Área de viviendas (Línea A y B)

- Cimientos de la casa

- Erguimiento de paredes en madera

- Instalaciones de ventanales y puertas

- Colocación del mediotecho

- Colocación de los paneles solares fotovoltaicos

- Colocación de la palmicha para la techumbre lateral

- Acondicionamiento del sistema eléctrico fotovoltaico

- Instalación de los tanques de recepción hídrica

- Instalación de las baterías sanitarias, duchas, lavabos y lavadero

- Instalación de locetas y paneles de cocina

\section{Acabados}

Se realizarán los acabados de pintura, cielo raso y marcos para cada ecovivienda

\section{Red de Agua Potable:}

Se realizará la red de agua potable primaria, instalación contra incendio y red de aguas negras.

\section{Obras Exteriores:}




\section{GESTIÓN DE LOS RECURSOS HUMANOS}

Esta parte que corresponde a la planificación de recursos humanos se basa en los requisitos de los recursos de las actividades para determinar las necesidades de recursos humanos para el proyecto según la guía PMBOK (PMI, 2008). La obra contara con un equipo de proyecto que dirija el mismo y complemente con la empresa tercerizada (Consorcio Kaeko)

\section{Planificación de los Recursos Humanos}

\section{Factores Técnicos dentro del proyecto}

Se creará un grupo interdisciplinario para la gestión planificación y ejecución del proyecto, cabe anotarse que como se piensa tercerizar esta labor, la potestad de escogencia del personal calificado y no calificado la tiene el Consorcio, en tal sentido se presume que ellos cuentan con su propio arquitecto, un ingeniero estructural, un ingeniero mecánico y un ingeniero eléctrico. En lo que concierne al estudio de factibilidad si se requerirá de un consultor metodológico que puede ser un Ingeniero Industrial, un Administrador de Empresas, o un Profesional de Mercados, y para lo correspondiente a la medición de distancias respecto a tierras y edificaciones se requerirá de un topógrafo, pero de igual forma, es el Consorcio tercerizado Se contará eso sí, con un director de proyectos para toda la obra, pero el interactuará conforme a los lineamientos del Consorcio.

\section{Factores Interpersonales dentro del proyecto}

Como se contratará una empresa vía Outsourcing, este apartado de los factores interpersonales es competencia de este consorcio con injerencia solamente del Director del proyecto.

\section{Plantillas de Recursos Humanos y Listas de Control}


Estas plantillas de recursos humanos y listas de control tomarán como fundamento el organigrama del proyecto, la descripción de roles y responsabilidades, la evaluación de empresas ejecutantes (Consorcio Kaeko) y los lineamientos de Seguridad y Salud en el Trabajo, según el Decreto 1443 de 2014.

\section{Adquirir el Equipo de Proyecto}

\section{Roles y Responsabilidades}

Con la intencionalidad de cumplir con los objetivos trazados, se determinan los siguientes roles y responsabilidades dentro del equipo de proyecto.

\section{Patrocinador del Proyecto}

Como representante legal, se nombrará el Comerciante José Armando Velásquez, quien aparte de financiar el proyecto velará porque no esta obra llegue a buen término.

\section{- Director del Proyecto}

El Arquitecto Samuel Arango Shima, es la persona que será responsable de identificar los requisitos, establecer objetivos claros y posibles de realizar, equilibrar las demandas de calidad, alcance, tiempo y costos, recursos humanos y adquisiciones necesarias para la obra. El tendrá a cargo el grupo interdisciplinario del proyecto (topógrafo, ingeniero mecanic, contador, diseñador, entre otros)

\section{- Equipo de Proyecto}

El equipo de proyecto estará integrado por todos los colaboradores que estarán encargados del trabajo de construcción, el Director de Proyectos, la Constructora (Consorcio Kaeko) y el Patrocinador. El equipo de proyectos se identifica en el organigrama.

\section{- Empresa Constructora}

Este grupo (Consorcio Kaeko) estará a cargo de la ejecución del proyecto y tendrá como responsabilidad realizar las actividades descritas en la EDT, clasificadas por tipode obra, en un tiempo y costo acordado y mediante un contrato firmado previamente.

Cuadro 2. Matriz de roles y responsabilidad del Equipo del Proyecto

\begin{tabular}{|c|c|c|c|c|c|}
\hline \multicolumn{5}{|c|}{ MATRIZ DE ROLES Y RESPONSABILIDADES } & Convenciones \\
\hline ACTIVIDAD DEL EDT & Patrocinador & $\begin{array}{l}\text { Director } \\
\text { del } \\
\text { Proyecto }\end{array}$ & $\begin{array}{l}\text { Equipo } \\
\text { interdisciplinario }\end{array}$ & Constructora & $\begin{array}{l}E=\text { Ejecutar/Elaborar } \\
P=\text { Participar } \\
C=\text { Coordinar } \\
R=\text { Revisar } \\
A=\text { Autorizar }\end{array}$ \\
\hline
\end{tabular}

APP

Entregable de la AP

ESTUDIO

PRELIMINAR 


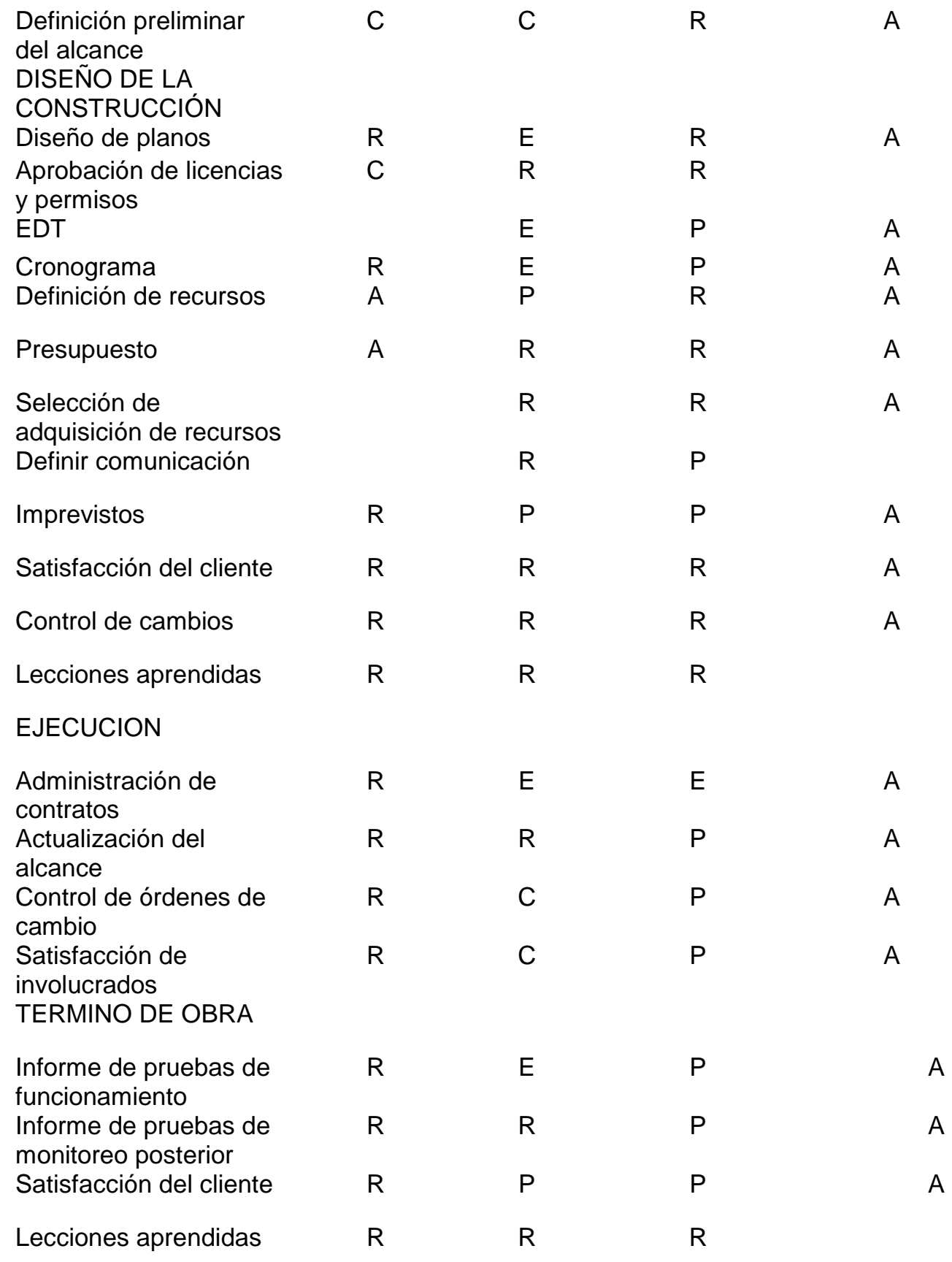

\section{Organigrama}

Figura 2. Organigrama del proyecto 


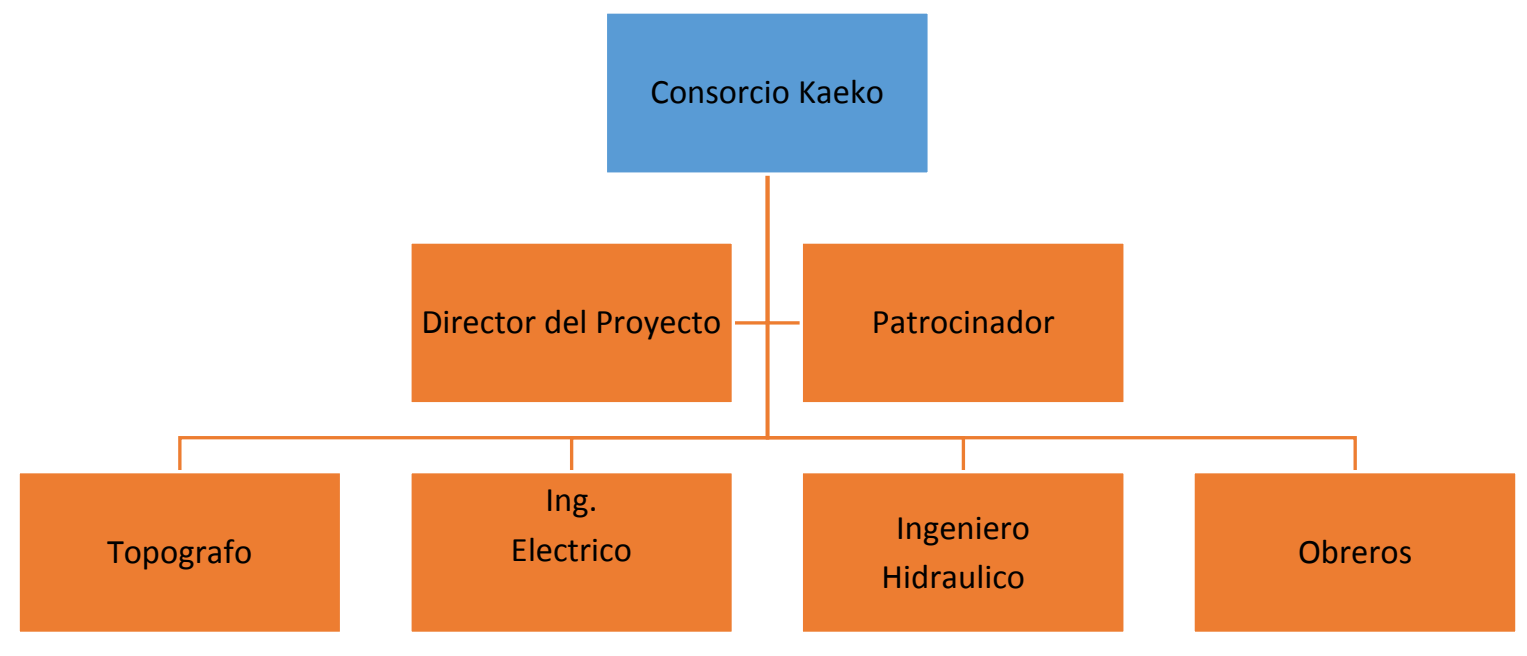

\section{Desarrollar Equipo de Proyecto}

\section{Adquisición y disponibilidad del personal del proyecto}

La disponibilidad del equipo de proyecto no es muy vasta, teniendo en cuenta que las construcciones ecosostenibles es un tema si se quiere nuevo en Colombia, no obstante es un tipo de construcción que tiene gran acogida por su representatividad para la conservación del medio ambiente. De todas formas, es necesario dada la relevancia social del proyecto objeto de estudio garantizar que el personal del proyecto, sea lo más idóneo posible.

Cuadro 3. Perfiles del equipo del proyecto

\begin{tabular}{ccc}
\hline Recurso humano asociado & Cantidad & \multicolumn{1}{c}{ Perfil } \\
\hline Director del Proyecto & 1 & $\begin{array}{l}\text { Profesional en Arquitectura, } \\
\text { preferiblemente, Ingeniero de } \\
\text { Proyectos, Ingeniero Industrial } \\
\text { Entidad con experiencia en } \\
\text { Constructora }\end{array}$ prostos de construcción, $^{\text {preferiblemente en ecotectura y }}$
\end{tabular}


Topógrafo

Ingeniero Eléctrico

Ingeniero Hidráulico

Obreros edificaciones ecosostenibles

Profesional en Topografia, con experiencia de màs de 3 años en proyectos de este estilo Profesional con experiencia en tendidos eléctricos y conocimiento en instalaciones de paneles solares fotovoltaicos Profesional en ingeniería hidráulica con experiencia de màs de 3 años en este tipo de proyectos

Bachilleres o técnicos de construcción, con experiencia de más de 3 años en construcciones

\section{Seguridad y salud en el Trabajo}

Teniendo en cuenta la implementación del SGSST o el nivel del mismo que tenga la empresa (Consorcio Kaeko) se deberán tener en cuenta las siguientes recomendaciones

- Protocolo de trabajo en alturas

- Riesgos eléctricos (instalación de paneles fotovoltaicos)

- Riesgos biomecánicos

- Riesgos físicos

- Riesgos biológicos

- Uso de equipos de protección asociados

\section{GESTIÓN DE COMUNICACIONES}

\section{Entradas del Plan de Gestión de Comunicaciones}

\section{Acta de Constitución del Proyecto}

Este documento se muestra en un que acta deberá ser revisada y firmada previamente a la iniciación del proyecto4.4.1. Planificación de las Comunicaciones

\section{Tecnología de la Información}

Para este proyecto se promoverán se combinarán medio de comunicación tradicionales (como el teléfono, radio teléfonos) con medios virtuales (Internet, smartphones, tablets, etc) con el fin de garantizar el flujo efectivo de la información, con sus respectivos informes físicos o virtuales. Se propondrán reuniones semanales, 
donde se aclararán dudas, solicitudes y aprobación de órdenes de cambio, riesgos y posibles atrasos o alteraciones al presupuesto, informes de rendimientos, imprevisto general y omisiones en los planos del proyecto.

El Director del Proyecto deberá presentar un informe mensual al patrocinador y un representante del Consorcio donde deberá incluir; solicitudes de cambio, criterios de desempeño del proyecto.

\section{Resultados y Discusión}

Un proyecto como este constituye un excelente precedente porque las comunidades en el último tiempo quieren participar activamente en la conservación del planeta, además del aprovechamiento de los recursos en función del desarrollo sostenible mismo.

Económicamente, un proyecto como este, es de gran aceptación desde la óptica de la relación costo/beneficio, porque por ejemplo, con las instalaciones de paneles solares fotovoltaicos, se ahorra un $95 \%$ en costos de energía tradicional, lo mismo sucede con el aprovechamiento de las aguas lluvias, y de las aguas naturales de las sequias rurales donde se va a construir el condominio.

Socialmente, el proyecto de ecoviviendas, pone un paradigma diferente en cuanto a la posibilidad del cambio de pensamiento, puesto que la gente del común, va ganando en una convivencia más sana con su entorno, va adquiriendo un rol menos perjudicial y más comprometido con las generaciones venideras.

\section{Conclusiones}

Por medio de la realización de la presente investigación se consideraron diferentes Planes de Gestión los cuales cada uno resultaba necesario para el éxito y progreso del proyecto; en este orden de ideas se pudo evidenciar que entre más detalladas sean las actividades de cada plan, màs cercano se encuentra el logro del objetivo trazado.

De igual forma, se puedo constatar, que los proyectos no tienen estándares fijos, dicho de otra manera se pudo evidenciar en este proyecto, que ningún proyecto se parece a otro, aun tengan características similares.

Con la realización del Plan de Gestión del Alcance realizado por ejemplo, se pudo corroborar con claridad que los proyectos dependerán de lo que se planifique, antes que lo que se modifique sobre la marcha. Ningún proyecto cumple con precisión los parámetros preestablecidos, pero no es menos cierto manifestar que ningún proyecto puede tener éxito si no se toman las instancias preliminares pertinentes.

\section{Literatura citada}

López Doval, E. (2012). El proyecto del coche eléctrico en España. La gestión basada en PMBOK. Madrid: Escuela Universitaria Gimbernat y Tomàs Cerdà. 
PMI. (2008). Guia de los fundamentos de administracion de proyectos (Quinta Edición ed.).

Ramirez Zuluaga, C. (2011). Integración entre PSP y PMBOK aplicada al desarrollo de un sistema experto para el diagnóstico e identificación automática de enfermedades profesionales. Manizales.

Soto Vicente, E. (2015). Aplicación de la guía del PMBOK en el desarrollo de nuevos productos farmaceúticos en un área de investigación y desarrollo. Lima. 